\title{
Some Remarks on the Lax-Wendroff Finite-Difference Scheme for Nonsymmetric Hyperbolic Systems*
}

\author{
By Masaya Yamaguti
}

1. Let us consider the following system of equations:

$$
\frac{\partial u}{\partial t}=\sum_{k=1}^{n} A_{k} \frac{\partial u}{\partial x_{k}}
$$

where $u$ is an unknown $N$-vector, $A_{k}$ are real constant $N$ by $N$ matrices. It is well known that the Cauchy problem for (1) with initial data

(2) $u(x, 0)=u_{0}(x)$ is well posed in the $L^{2}$ sense if we assume the matrices $A_{k}$ to be symmetric. We know also that there is another class of systems for which we can prove the well posedness of the Cauchy problem in the $L^{2}$ sense. That class is defined by the condition:

(3) all eigenvalues of $\sum_{k=1}^{n} A_{k} \xi_{k}$ are always real and distinct for all real $\xi,|\xi| \neq 0$, and such equations are called a strictly hyperbolic system or a regularly hyperbolic system.

In [1], Peter Lax remarked that there are systems in the class of strictly hyperbolic equations for which we can not symmetrize all $A_{k}$ by multiplying with one constant symmetric positive matrix. At the same time he remarked that the symmetric hyperbolic system is very sensitive to small perturbation of the $A_{k}$, but strictly hyperbolic systems obviously are not sensitive to such perturbations. For the numerical solution of the Cauchy problem, we know that many finite-difference schemes work nicely for the symmetric hyperbolic system. Among them, Friedrichs' scheme, which is accurate of first order, is stable for sufficiently small mesh ratio (the ratio is determined by the Courant-Friedrichs-Lewy condition) for the above two classes of hyperbolic systems, namely the symmetric or the strictly hyperbolic system. This is proved in [1]. On the other hand, the Lax-Wendroff scheme which has second-order accuracy was proved stable if the mesh ratio is small, for any symmetric hyperbolic system [3]. Now, in this paper we are going to point out that for the nonsymmetric hyperbolic system, even for the strictly hyperbolic system, the Lax-Wendroff scheme is not always stable, if $n>1, N>2$, no matter how small the mesh ratio is. (We can trivially prove that if $n=1$ or $N \leqq 2$, the scheme is always stable for small mesh ratio.) Also we derive a criterion that assures the stability of the Lax-Wendroff scheme.**

Received December 19, 1966. Revised April 5, 1967.

* The research for this paper was performed at the Courant Institute of Mathematical Sciences, New York University, under NSF grant No. GP-3465, during the academic year 1964-1965.

** P. D. Lax has notified me that Burton Wendroff recently showed that a modified LaxWendroff scheme (modified in the sense of Richtmyer), with a reasonsable mesh ratio, is stable for the regularly hyperbolic system. Wendroff's manuscript [8], kindly sent to me by the author, indicates that the scheme is quite efficient and stable for symmetric systems as well as regularly hyperbolic systems. 
2. We discuss only the case $n=2$, but remark that the general case can be treated in the same fashion. Consider the Lax-Wendroff scheme for the system (1) for $n=2$;

$$
\begin{aligned}
& \frac{\partial u}{\partial t}=A \frac{\partial u}{\partial x}+B \frac{\partial u}{\partial y}: u(x, y, t+h) \\
& =\left\{I+\lambda\left(A D_{0 x}+B D_{0 y}\right)\right. \\
& \left.+\frac{\lambda^{2}}{2}\left(A^{2} D_{+x} D_{-x}+(A B+B A) D_{0 x} D_{0 y}+B^{2} D_{+y} D_{-y}\right)\right\} u(x, y, t)
\end{aligned}
$$

where the operators $D_{0 x}, D_{0 y}, D_{ \pm x}, D_{ \pm y}$ are defined in the following way:

$$
\begin{aligned}
2 D_{0 x} u(x, y) & =u(x+k, y)-u(x-k, y) \\
2 D_{0 y} u(x, y) & =u(x, y+k)-u(x, y-k) \\
D_{+x} u(x, y) & =u(x+k, y)-u(x, y) \\
D_{+y} u(x, y) & =u(x, y+k)-u(x, y) \\
D_{-x} u(x, y) & =u(x, y)-u(x-k, y) \\
D_{-y} u(x, y) & =u(x, y)-u(x, y-k)
\end{aligned}
$$

and $\lambda=h / k$.

Upon taking the Fourier transform, we compute the amplification matrix (see [2]) of the scheme:

$$
\begin{aligned}
c(\xi, \eta)= & I+i \lambda(A \sin \xi+B \sin \eta) \\
& -\lambda^{2}\left\{A^{2}(1-\cos \xi)+\frac{1}{2}(A B+B A) \sin \xi \sin \eta+B^{2}(1-\cos \eta)\right\} \\
= & I+i \lambda(A \sin \xi+B \sin \eta)-\frac{\lambda^{2}}{2}(A \sin \xi+B \sin \eta)^{2} \\
& -2 \lambda^{2}\left(A^{2} \sin ^{4} \frac{\xi}{2}+B^{2} \sin ^{4} \frac{\eta}{2}\right) .
\end{aligned}
$$

It is well known (see [2]) that the von Neumann condition is necessary for the stability of (4). Hence we only have to check that the absolute value of some eigenvalue of this matrix is greater than one (a violation of the von Neumann condition), in order to show the instability of this scheme. We exhibit this in the following two examples.

Example 1.

$$
A_{0}=\left[\begin{array}{rrr}
1 & 0 & 0 \\
0 & -1 & 0 \\
0 & 0 & 0
\end{array}\right], \quad B_{0}=\left[\begin{array}{rrr}
0 & 1 & \epsilon \\
1 & 0 & 0 \\
-\epsilon & 0 & 0
\end{array}\right], \quad \epsilon>0 .
$$

Here $\epsilon$ is a small positive number.

We can show very easily the following facts:

(i) For any real $(\xi, \eta), A_{0} \xi+B_{0} \eta$ has three real distinct roots. (Of course, it is necessary to take $\epsilon$ sufficiently small.)

(ii) $A_{0}$ and $B_{0}$ can not be symmetrized simultaneously by multiplication with a positive definite matrix. 
(iii) For any positive numbers $(\chi, \zeta), A_{0}^{2} \chi+B_{0}^{2} \zeta$ has one negative eigenvalue. We simply prove (iii).

$$
A_{0}{ }^{2} \chi+B_{0}{ }^{2} \zeta=\left[\begin{array}{ccc}
\chi+\left(1-\epsilon^{2}\right) \zeta & 0 & 0 \\
0 & \zeta+\chi & \epsilon \zeta \\
0 & -\epsilon \zeta & -\epsilon^{2} \zeta
\end{array}\right],
$$

$\operatorname{det}\left(A_{0}{ }^{2} \chi+B_{0}{ }^{2} \zeta\right)=\left\{\chi+\left(1-\epsilon^{2}\right) \zeta\right\}\left(-\epsilon^{2} \zeta \chi\right)<0$.

But the determinant of a matrix is equal to the product of all its eigenvalues, therefore, (iii) follows since any complex eigenvalues constitute a conjugate complex pair. Now let us consider the matrix $c(\xi, \eta)$ at $\xi=\pi, \eta=\pi$. We have

$$
c(\pi, \pi)=I-2 \lambda^{2}\left(A_{0}{ }^{2}+B_{0}{ }^{2}\right) .
$$

One eigenvalue of this matrix is greater than 1 for any $\lambda \neq 0$.

This proves the instability of the scheme for the strictly hyperbolic system

$$
\frac{\partial u}{\partial t}=A_{0} \frac{\partial u}{\partial x}+B_{0} \frac{\partial u}{\partial y} .
$$

Remark 1. It is interesting to see that the instability occurs for the highest fre. quencies (neighborhood of $\pi$ ).

Remark 2. Both matrices $A_{0}$ and $B_{0}$ have a zero eigenvalue.

Example 2.

$$
A_{1}=\left[\begin{array}{rrr}
p & 0 & 0 \\
0 & -p & 0 \\
0 & 0 & 0
\end{array}\right], \quad B_{1}=\left[\begin{array}{rrr}
0 & m & 1 \\
k & 0 & 1 \\
-1 & -1 & 0
\end{array}\right],
$$

where $k \neq \pm m$ and $k, m$ satisfy the following condition:

$$
4(m k-2)^{3}-27(m+k)^{2}>0 .
$$

If we take $p>0$ and sufficiently large, then we can show the instability of (4) with $A \equiv A_{1}$ and $B \equiv B_{1}$. First we show the strict hyperbolicity of $A \xi+B \eta$ for real $\xi, \eta$. It is easy to verify that $B_{1}$ has no zero eigenvalue. The characteristic equation of $A \xi+B \eta$ is

$$
\operatorname{det}\left[\begin{array}{ccr}
p \xi-\mu & m \eta & \eta \\
k \eta & -p \xi-\mu & \eta \\
-\eta & -\eta & -\mu
\end{array}\right]=0
$$

that is,

$$
\mu^{3}-\left\{p^{2} \xi^{2}+(m k-2) \eta^{2}\right\} \mu+(m+k) \eta^{3}=0 .
$$

Taking the discriminant $D$ of this equation, we have

$$
\begin{aligned}
D & =-4\left\{p^{2} \xi^{2}+(m k-2) \eta^{2}\right\}^{3}+27(m+k)^{2} \eta^{6} \\
= & -\eta^{6}\left\{4 p^{6} x^{3}+12 p^{4}(m k-2) x^{2}+12 p^{2}(m k-2)^{2} x\right. \\
& \left.\quad+4(m k-2)^{3}-27(m+k)^{2}\right\} \\
= & -\eta^{6} P(x),
\end{aligned}
$$

where $x=\xi^{2} / \eta^{2}$. We see that $4(m k-2)^{3}-27(m+k)^{2}>0$ implies $P(x)>0$ for 
all $x>0$. Thus, we can conclude that $D$ is always negative, which means (8) has three real distinct roots.

Next, we show, as in Example 1, that $A_{1}{ }^{2}+B_{1}{ }^{2}$ has a negative real eigenvalue. We compute $A_{1}{ }^{2}+B_{1}{ }^{2}$ :

$$
A_{1}{ }^{2}+B_{1}{ }^{2}=\left[\begin{array}{ccr}
p^{2}+m k-1 & -1 & m \\
-1 & p^{2}+m k-1 & k \\
-k & -m & -2
\end{array}\right] .
$$

Consider the equation

$$
-\operatorname{det}\left[A_{1}^{2}+B_{1}^{2}\right]=2 p^{4}+2 p^{2}(m k-2)-2 m k-m^{2}-k^{2}=0 .
$$

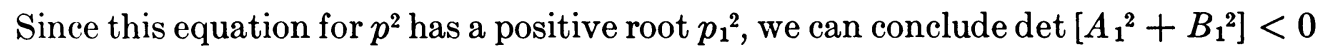
for large $p^{2}$. Therefore, the matrix $A_{1}{ }^{2}+B_{1}{ }^{2}$ has one negative eigenvalue. Hence, as before, by substitution in (6) we see that (4) is unstable.

3. Conditions that Assure the Stability of Finite-Difference Schemes for a Strictly Hyperbolic System. In this paragraph, we review the definition of stability given by Lax-Richtmyer for finite-difference schemes for solving a strictly hyperbolic system (1). First, we introduce the finite-difference scheme for solving the Cauchy problem. We consider the operator $S_{k}$ in ${L_{x}}^{2}$ defined by the following:

$$
\begin{gathered}
u(x, t+h)=S_{k} u(x, t), \quad x=\left(x_{1}, \cdots, x_{n}\right), \\
S_{k}=\sum_{j} c_{j} T^{j},
\end{gathered}
$$

where $j$ is a multi-index $\left(j_{1}, \cdots, j_{n}\right)$, and $T^{j}$ represents a product of translation operators $T_{1}{ }^{j_{1}}, \cdots, T_{n}{ }_{n}$. $\left(T_{i}\right.$ is defined by $T_{i} u(x)=u\left(x+k e_{i}\right)$, where $e_{i}$ is the unit vector, $e_{i}=\left(\delta_{i k}\right), 1 \leqq k \leqq n$.) Of course, we denote by $h$ the time mesh-length, and by $k$ the space mesh-length. We obtain the amplification matrix by taking the Fourier transform:

$$
c(\xi)=\sum_{j} c_{j} \exp \left[i j^{\xi}\right], \quad \xi=\left(\xi_{1}, \cdots, \xi_{n}\right) .
$$

As stated in [2], Lax-Richtmyer's definition of stability is equivalent to the requirement that $c^{n}(\xi)$ is uniformly bounded for all integral $n\left(\left|\xi_{i}\right| \leqq \pi\right)$. There are many practical criteria that assure this stability. Before introducing such criteria, we explain some notions which are useful (see [3], [5]).

Definition. We say the scheme (11) has "accuracy of order $p$ " when (12) satisfies the following inequality for small $\xi$, with nonnegative integer $p$, and constant $K$ :

$$
|\exp [i \lambda A \cdot \xi]-c(\xi)| \leqq K|\xi|^{p+1}
$$

where $A \cdot \xi$ signifies $\sum_{k=1}^{n} A_{k} \xi_{k}, \lambda=h / k,|\xi|^{2}=\xi_{1}{ }^{2}+\xi_{2}{ }^{2}+\cdots+\xi_{n}{ }^{2}$, and $|A|$ is the natural matrix norm defined by $|A|=\sup _{|x| \neq 0}|A x| /|x|$.

Definition. We say the scheme (11) has "dissipation of degree $2 r$ " when all the eigenvalues $k_{p}(\xi)$ of $c(\xi)$ in (12) satisfy the following inequality with some constant $\delta>0$ and positive integer $r$ :

$$
\left|k_{p}(\xi)\right| \leqq 1-\delta|\xi|^{2 r} \quad\left(\left|\xi_{i}\right| \leqq \pi,(i=1,2, \cdots, n)\right)(p=1, \cdots, N)
$$


Using this terminology, some practical conditions which imply Lax-Richtmyer stability are given in the following two theorems.

TheOREM 1. For a strongly hyperbolic system (1), if the scheme (11) has accuracy of order $2 p-1$ and dissipation of degree $2 p$, then the scheme is stable (Kreiss [4]).

For the definition of a strongly hyperbolic system, see [6], [7]. We only say that a strictly hyperbolic system is strongly hyperbolic.

Theorem 2. For a symmetric hyperbolic system (1), if the scheme (11) has Hermitian coefficients and has accuracy of order $2 p-2$ and dissipation of degree $2 p$, then it is stable (Parlett [5]).

We now prove the following theorem which gives a new criterion for assuring stability.

Theorem 3. For a strictly hyperbolic system (1), if scheme (11) has accuracy greater than 1 and if it satisfies one of the two following conditions, then it is stable:

(i) $c(\xi)$ has dissipation of some degree $2 r>0$, for $\xi \neq 0$;

(ii) the eigenvalues of $c(\xi)$ are distinct for all $\xi \neq 0$ and the von Neumann condition is satisfied.

Proof. First we prove that $c(\xi)$ is uniformly diagonalizable in a sufficiently small neighborhood of $\xi=0$. Observe that $c(0)=I$, and for small $|\xi|$,

$$
\log c(\xi)=\frac{c(\xi)-I}{1}-\frac{(c(\xi)-I)^{2}}{2}+\cdots
$$

Therefore, since the scheme has accuracy of order greater than 1, (13) yields

$$
\log c(\xi)=i \lambda A \cdot \xi+R(\xi), \quad|R(\xi)|=O\left(|\xi|^{p}\right), \quad p>1 .
$$

If we put $|\xi|=\rho, \xi /|x i|=\xi^{\prime}$, then we have for $\rho \neq 0$

$$
\log c(\xi) / \rho=i \lambda A \cdot \xi^{\prime}+R_{1}\left(\rho, \xi^{\prime}\right), \quad\left|R_{1}\left(\rho, \xi^{\prime}\right)\right|=O\left(\rho^{p-1}\right) .
$$

Since the eigenvalues of $A \cdot \xi^{\prime}$ are distinct on $\left|\xi^{\prime}\right|=1$, by the strict hyperbolicity, the eigenvalues of $\log c(\xi) / \rho$ are also distinct (uniformly) for small $\rho$. Thus, we can say there is a positive number $\gamma$ such that $\log c(\xi) / \rho$ is "uniformly diagonalizable" for $\rho \leqq \gamma$. The uniform diagonalizability is defined as follows: When $X$ is the matrix whose columns are a complete set of unit eigenvectors of the matrix $E$, then the diagonal matrix of eigenvalues, $\Lambda$, is given by

$$
\Lambda=X^{-1} E X .
$$

We say the family $E(\xi)$ is uniformly diagonalizable if $[X(\xi)]^{-1}$ is uniformly bounded. Since $\operatorname{det} X\left(\xi^{\prime}\right)$, for $E \equiv \log c(\xi) / \zeta$, is a continuous nonzero function on $\left|\xi^{\prime}\right|=1$, Weierstrass' theorem implies $|\operatorname{det} X| \geqq \mu>0$, for some constant $\mu$. Hence $X^{-1}$ is uniformly bounded. This implies that $c(\xi)$ is also uniformly diagonalizable for $\rho \leqq \gamma$. Before completing the proof of stability, we observe that if the scheme satisfies the condition (ii), then it is easy to see that $c(\xi)$ is uniformly diagonalizable for all $\xi,\left|\xi_{i}\right| \leqq \pi$, and the von Neumann condition is satisfied, which implies the stability by the discussion of Lax-Richtmyer [2].

Let us now return to case (i). We can limit consideration to the range $\left|\xi_{i}\right| \leqq \pi$, $\gamma \leqq \rho$ by the periodicity of $c(\xi)$ and by the above proof for the range $\rho \leqq \gamma$. By taking a suitable unitary matrix $U(\xi)$, we can transform $c(\xi)$ to an upper triangular matrix, $B(\xi)$, 


$$
B(\xi)=U(\xi) c(\xi) U^{*}(\xi)=K(\xi)+B_{1}(\xi),
$$

where $K(\xi)$ is a diagonal matrix and $B_{1}(\xi)$ is a strictly upper triangular matrix. Of course, the diagonal elements of $K(\xi)$ are the eigenvalues of $c(\xi)$. Now with a diagonal matrix $D$ of the form:

$$
D=\left[\begin{array}{llllll}
d & & & & & \\
& & & & \\
& & d^{2} & & & \\
& & & \cdot & \\
& & & d^{n}
\end{array}\right],
$$

we transform $B$ to $D B D^{-1}=K+B_{2}$ with

$$
B_{2}=\left[\begin{array}{cccccc}
0 & \frac{b_{12}}{d} & \cdot & \cdot & \cdot & \frac{b_{1 n}}{d^{n-1}} \\
\vdots & & \cdot & \cdot & \cdot \\
\vdots & & & & \frac{b_{n-1 n}}{d} \\
0 & & & . & . & 0
\end{array}\right] .
$$

By the condition (i), we have

$$
K^{*} K \leqq\left(1-\delta|\xi|^{2 r}\right) I
$$

Taking $d$ large, we have

$$
\left(K+B_{2}\right) *\left(K+B_{2}\right) \leqq I
$$

which means that all eigenvalues of $\left(K+B_{2}\right)^{*}\left(K+B_{2}\right)$ are less than 1 , since $\left(K+B_{2}\right)^{*}\left(K+B_{2}\right)=K^{*} K+K^{*} B_{2}+B_{2}{ }^{*} K+B_{2}{ }^{*} B_{2}$ and $\rho=|\xi| \geqq \gamma$. Therefore, if we put $T_{2}=D U$, there is a positive constant $\alpha$ such that

$$
\max \left(\left|T_{2}\right|,\left|T_{2}\right|^{-1}\right) \leqq \alpha \text { and }\left|T_{2} c T_{2}{ }^{-1}\right| \leqq 1,
$$

where we use the natural matrix norm $|A|=\sup _{x \neq 0}|A x| /|x|$ corresponding to the Euclidean norm $|x|$. Therefore, all powers of $c$ satisfy $\left|T_{2} c^{n} T_{2}{ }^{-1}\right| \leqq 1$, whence $\left|c^{n}\right| \leqq \alpha^{2}$ and are bounded.

\section{The Lax-Wendroff Scheme for a Strict Hyperbolic System.}

$$
\frac{\partial u}{\partial t}=A \frac{\partial u}{\partial x}+B \frac{\partial u}{\partial y} .
$$

We derive a sufficient condition for the stability of the Lax-Wendroff scheme for special strictly hyperbolic systems. We express the condition by using the following notation.

$$
\begin{aligned}
& A_{0}(\xi, \eta)=A \sin \xi+B \sin \eta, \\
& A_{1}(\xi, \eta)=A^{2} \sin ^{4} \xi / 2+B^{2} \sin ^{4} \eta / 2 .
\end{aligned}
$$

TheOREM 4. The Lax-Wendroff scheme is stable for sufficiently small mesh ratio $\lambda$, if the coefficient matrices $A, B$ of the strictly hyperbolic system satisfy the following conditions: 
(1) at least one of $A$ and $B$ does not have a zero eigenvalue;

(2) $A^{2} \xi+B^{2} \eta$ has only real eigenvalues for all real $\xi, \eta$;

(3) for each eigenvalue of $A_{0}(\xi, \eta)$ we denote by $\phi_{0}$ and $\psi_{0}$ the eigenvectors corresponding to this eigenvalue of $A_{0}(\xi, \eta)$ and $A_{0}{ }^{*}(\xi, \eta)$ respectively, such that $\left\langle\phi_{0}, \psi_{0}\right\rangle>0$; we have

$$
\left\langle A_{1}(\xi, \eta) \phi_{0}, \psi_{0}\right\rangle \geqq l_{\rho}^{4}\left\langle\phi_{0}, \psi_{0}\right\rangle
$$

with $l$ being a positive constant and $\rho^{2}=\sin ^{2} \xi+\sin ^{2} \eta$.

Proof. First we remark that the matrix $A_{0}(\xi, \eta)$ vanishes at $(\pi, \pi)$ but $A_{1}(\xi, \eta)$ does not vanish there. To treat this case specially, we divide our discussion into two parts. Namely, for $(\xi, \eta)$ which satisfy $\pi-\epsilon \leqq|\xi| \leqq \pi, \pi-\epsilon \leqq|\eta| \leqq \pi$, and for $(\xi, \eta)$ which do not satisfy these inequalities.

(i) For $(\xi, \eta)$ which satisfy $\pi-\epsilon \leqq|\xi| \leqq \pi, \pi-\epsilon \leqq|\eta| \leqq \pi$, by assumption (2) and by Lax's concavity theorem for hyperbolic matrices [1], we can show that the eigenvalues $a_{p}(\xi, \eta)$ of

$$
A_{1}(\xi, \eta)=A^{2} \sin ^{4} \xi / 2+B^{2} \sin ^{4} \eta / 2 \quad(p=1,2, \cdots, N)
$$

are strictly positive, that is to say, bounded below by some positive constant which is independent of $\epsilon$ and $(\xi, \eta)$ for this range. Therefore, the eigenvalues $k_{p}(\xi, \eta)$ have the following form:

$$
k_{p}(\xi, \eta)=1-a_{p}(\xi, \eta)+O(\epsilon) \quad(p=1,2, \cdots, N)
$$

which satisfy the condition (14) for sufficiently small $\epsilon$. This proves that condition (i) of Theorem 4 is satisfied for this range.

(ii) Now we discuss the domain of $\xi, \eta$ except the neighborhood of $|\xi|=\pi$, $|\eta|=\pi$. We use condition (3) to prove some dissipation stated in condition (i) for small $\lambda$. Putting $\rho^{2}=\sin ^{2} \xi+\sin ^{2} \eta, \sin \xi / \rho=\xi^{\prime}, \sin \eta / \rho=\eta^{\prime}\left(\xi^{\prime 2}+\eta^{\prime 2}=1\right)$ and $A_{1}(\xi, \eta) / \rho=A_{1}{ }^{\prime}(\xi, \eta)$, we consider the matrix

$$
c_{1}(\xi, \eta)=i A_{0}\left(\xi^{\prime}, \eta^{\prime}\right)+(-1) \lambda \frac{\rho}{2} A_{0}\left(\xi^{\prime}, \eta^{\prime}\right)^{2}+2 A_{1}^{\prime}(\xi, \eta)
$$

as a perturbation of the matrix $A_{o}\left(\xi^{\prime}, \eta^{\prime}\right)$ by taking $\lambda$ small. We know that by the strict hyperbolicity, the absolute value of the difference of any two eigenvalues of $A_{0}\left(\xi^{\prime}, \eta^{\prime}\right)$ is bounded away from zero for $\xi^{\prime 2}+\eta^{\prime 2}=1$. Then we can consider the convergent Taylor expansion in $\lambda$ of an eigenvalue of $c_{1}(\xi, \eta)$.

In the following, we carry out this expansion. Let $\nu(\xi, \eta)$ be an eigenvalue of $c_{1}(\xi, \eta)$, and $\phi(\xi, \eta)$ be an eigenvector. Then we have

$$
\begin{aligned}
& \nu(\xi, \eta)=\nu_{0}+\lambda \nu_{1}+\lambda^{2} \nu_{2}+\cdots, \\
& \phi(\xi, \eta)=\phi_{0}+\lambda \phi_{1}+\lambda^{2} \phi_{2}+\cdots ;
\end{aligned}
$$

substituting these in $c_{1}(\xi, \eta) \phi=\nu \phi$, we obtain

$$
\begin{array}{r}
{\left[i A_{0}\left(\xi^{\prime}, \eta^{\prime}\right)-\lambda\left(\frac{\rho}{2} A_{0}\left(\xi^{\prime}, \eta^{\prime}\right)^{2}+2 A_{1}^{\prime}(\xi, \eta)\right)\right]\left(\phi_{0}+\lambda \phi_{1}+\cdots\right)} \\
=\left(\nu_{0}+\lambda \nu_{1}+\cdots\right)\left(\phi_{0}+\lambda \phi_{1}+\cdots\right) .
\end{array}
$$

By equating the coefficients of $\lambda^{0}$, we have $i A_{0}\left(\xi^{\prime}, \eta^{\prime}\right) \phi_{0}=\nu_{0} \phi_{0}$. Therefore, if one of the eigenvalues of $A_{0}\left(\xi^{\prime}, \eta^{\prime}\right)$ is $\mu_{0}$, then we have $\nu_{0}=i \mu_{0}$, and $\phi_{0}$ is the corre- 
sponding eigenvector of $A_{0}$.

From the coefficients of the term $\lambda^{1}$, we have

$$
i A_{0}\left(\xi^{\prime}, \eta^{\prime}\right) \phi_{1}-\left[\frac{\rho}{2} A_{0}\left(\xi^{\prime}, \eta^{\prime}\right)^{2}+2 A_{1}^{\prime}(\xi, \eta)\right] \phi_{0}=i \mu_{0} \phi_{1}+\nu_{1} \phi_{0} .
$$

That is,

$$
i\left(A_{0}{ }^{\prime}\left(\xi^{\prime}, \eta^{\prime}\right)-\mu_{0} I\right) \phi_{1}=\left[\nu_{1} I+\frac{\rho}{2} A_{0}{ }^{\prime}\left(\xi^{\prime}, \eta^{\prime}\right)^{2}+2 A_{1}{ }^{\prime}(\xi, \eta)\right] \phi_{0} .
$$

We can determine $\phi_{1}$ by determining $\nu_{1}$ from the following equation, since the eigenvectors of a matrix $E$ and $E^{*}$ are biorthogonal:

$$
\begin{aligned}
& \nu_{1}=-\frac{\left\langle\left(\frac{\rho}{2} A_{0}{ }^{\prime}\left(\xi^{\prime}, \eta^{\prime}\right)^{2}+2 A_{1}{ }^{\prime}(\xi, \eta)\right) \phi_{0}, \psi_{0}\right\rangle}{\left\langle\phi_{0}, \psi_{0}\right\rangle} \\
&=-\frac{\rho}{2} \mu_{0}{ }^{2}-\frac{2\left\langle A_{1}{ }^{\prime}(\xi, \eta) \phi_{0}, \psi_{0}\right\rangle}{\left\langle\phi_{0}, \psi_{0}\right\rangle} .
\end{aligned}
$$

By assumption (3) we have

$$
S=\frac{\left\langle A_{1}{ }^{\prime}(\xi, \eta) \phi_{0}, \psi_{0}\right\rangle}{\left\langle\phi_{0}, \psi_{0}\right\rangle} \geqq l_{\rho}^{3}>0,
$$

and by solving the equation (17), we have a vector $\phi_{1}$ whose components are pure imaginary and are at most $O(\rho)$ for small $\rho$. Next, by equating the coefficients of $\lambda^{2}$ in (16), we obtain

$$
i\left(A_{0}^{\prime}\left(\xi^{\prime}, \eta^{\prime}\right)-\mu_{0} I\right) \phi_{2}=\nu_{1} \phi_{1}+\left(\frac{\rho}{2} A_{0}{ }^{\prime}\left(\xi^{\prime}, \eta^{\prime}\right)^{2}+2 A_{0}{ }^{\prime}(\xi, \eta)\right) \phi_{1}+\nu_{2} \phi_{0} .
$$

We can determine $\nu_{2}$ by the following equation:

$$
\nu_{2}=-\frac{\left\langle\left(\frac{\rho}{2} A_{0}{ }^{\prime}\left(\xi^{\prime}, \eta^{\prime}\right)^{2}+2 A_{1}^{\prime}(\xi, \eta)+\nu_{1}\right) \phi_{1}, \psi_{0}\right\rangle}{\left\langle\phi_{0}, \psi_{0}\right\rangle} .
$$

We note that $\nu_{2}$ is pure imaginary and is at most $O\left(\rho^{2}\right)$ for small $\rho$. Now set $\nu_{2}=i \nu_{2}{ }^{\prime}$. By a similar discussion, we have $\nu_{3}$ which is real, determined by (18) and

$$
\nu_{3}=-\frac{\left\langle\left(\frac{\rho}{2} A_{0}{ }^{\prime}\left(\xi^{\prime}, \eta^{\prime}\right)^{2}+2 A_{1}{ }^{\prime}(\xi, \eta)+\nu_{1}\right) \phi_{2}+\nu_{2} \phi_{1}, \psi_{0}\right\rangle}{\left\langle\phi_{0}, \psi_{0}\right\rangle} .
$$

We note that $\nu_{3}$ is at most $O\left(\rho^{3}\right)$ for small $\rho$. Finally, we have

$$
\nu=i \mu_{0}+\lambda\left(-\rho \mu_{0}{ }^{2} / 2-2 s\right)+i \lambda^{2} \nu_{2}+\lambda^{3} \nu_{3}+\cdots .
$$

Therefore, we obtain an expansion of $k(\xi, \eta)$, an eigenvalue of $c(\xi, \eta)$, by the homogeneity of $\nu$ and the definition (5):

$$
k(\xi, \eta)=1+i \lambda \mu_{0}(\xi, \eta)-\lambda^{2}\left(\mu_{0}^{2}(\xi, \eta) / 2+2 s \rho\right)+i \lambda^{3} \nu_{2}^{\prime} \rho+\lambda^{4} \nu_{3} \rho+\cdots .
$$

Now we compute its absolute value: 


$$
\begin{aligned}
|k(\xi, \eta)|^{2}= & {\left[1-\lambda^{2}\left(\mu_{0}{ }^{2} / 2+2 s \rho\right)+\lambda^{4} \nu_{3} \rho+\cdots\right]^{2} } \\
& +\left[\lambda \mu_{0}+\lambda^{3} \nu_{2}^{\prime} \rho+\cdots\right]^{2} \\
= & 1-4 \lambda^{2} k \rho+\lambda^{4} F+\cdots \\
F= & \left(\mu_{0}{ }^{2} / 2+2 s \rho\right)^{2}+2 \nu_{3} \rho+2 \mu_{0} \nu_{2}^{\prime} \rho
\end{aligned}
$$

where $F$ is a quantity of at most $O\left(\rho^{4}\right)$ for small $\rho$. We can assume $|F| \leqq F^{\prime} \rho^{4}$ ( $F^{\prime}$ is a constant) for $|\rho| \leqq \rho_{0}$. Now we use condition (3) which implies $s \rho \geqq l \rho^{4}$. Thus we take $\lambda$ such that

$$
\lambda^{2} \leqq 2 l / F^{\prime}
$$

Then we have

$$
\begin{aligned}
|k(\xi, \eta)|^{2} & \leqq 1-\lambda^{2} \rho^{4}\left(4 l-F^{\prime} \lambda^{2}\right)+\cdots, \\
& \leqq 1-2 \lambda^{2} \rho^{4} l+\cdots
\end{aligned}
$$

which yields a dissipation of degree 4 for $\rho \leqq \rho_{0}$. For $\rho \geqq \rho_{0}$, we have

$$
|k(\xi, \eta)|^{2} \leqq 1-4 \lambda^{2} l_{\rho_{0}}{ }^{4}+\lambda^{4} F+\cdots,
$$

and taking $\lambda$ sufficiently small, we have some dissipation for this range of $(\xi, \eta)$. These facts imply that condition (i) of Theorem 3 is satisfied. This completes the proof.

Kyoto University

Faculty of Engineering

Kyoto, Japan

1. P. D. LAx, "Differential equations, difference equations and matrix theory," Comm. Pure Appl. Math., v. 11, 1958, pp. 175-194. MR 20 \#4572.

2. P. D. Lax \& R. Richtmyer, "Survey of the stability of linear finite difference equations," Comm. Pure Appl. Math., v. 9, 1956, pp. 267-293. MR 18, 48.

3. P. D. LAX \& B. WendrofF, "Difference schemes for hyperbolic equations with high order of accuracy," Comm. Pure Appl. Math., v. 17, 1964, pp. 381-398. MR 30 \#722.

4. H. O. KREISs, "On difference approximations of the dissipative type for hyperbolic differential equations," Comm. Pure Appl. Math., v. 17, 1964, pp. 335-353. MR 29 \#4210.

5. B. PARlett, "Accuracy and dissipation in difference schemes," Comm. Pure Appl. Math., v. 19, 1966, pp. 111-123. MR 33 \#5141.

6. K. KaSAhara \& M. YAmaguti, "Strongly hyperbolic systems of linear partial differential equations with constant coefficients," Mem. Coll. Sci. Univ. Kyoto Ser. A Math., v. 33, 1960/61, pp. 1-23. MR $22 \# 9740$.

7. H. O. Kreiss, "Über sachgemässe Cauchyprobleme," Math. Scand., v. 13, 1963, pp. 109128. MR 29 \#6177.

8. B. Wendroff, "Well-posed problems and stable difference operators," 1967. (To appear.) 